\title{
Emerging roles of microglia cells in the regulation of adult neural stem cells
}

\author{
Eduardo Lira-Diaz ${ }^{1,2}$, Oscar Gonzalez-Perez ${ }^{1}$ \\ ${ }^{1}$ Laboratory of Neuroscience, School of Psychology, University of Colima, Colima 28040, Mexico. \\ ${ }^{2}$ Medical Sciences PhD Program, School of Medicine, University of Colima, Colima 28040, Mexico.
}

Correspondence to: Dr. Oscar Gonzalez-Perez, Laboratory of Neuroscience, School of Psychology, University of Colima, Av. Universidad 333, Colima 28040, Mexico. E-mail: osglez@ucol.mx

How to cite this article: Lira-Diaz E, Gonzalez-Perez O. Emerging roles of microglia cells in the regulation of adult neural stem cells. Neuroimmunol Neuroinflammation 2016;3:204-6.

\section{Article history: Received: 27-05-2016 Accepted: 17-08-2016 Published: 26-09-2016}

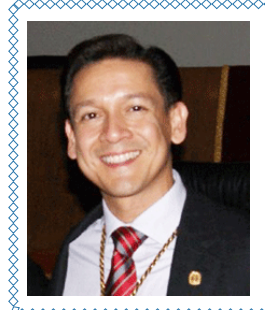

Dr. Oscar Gonzalez-Perez is Professor of Neuroscience in the School of Psychology at the University of Colima, Mexico (Research ID B-3680-2008, ORCID ID 0000-0002-6527-485X). He also is honorary professor of Neuroscience in the Doctorado en Ciencias Biomedicas at the University of Guadalajara, Mexico, and an invited professor of Neuroscience and Cellular Medicine in the Brain Tumor Stem Cell Laboratory of Dr. Alfredo Quinones-Hinojosa at Johns Hopkins University School of Medicine. He is also member of several editorial boards: Neuroimmunology and Neuroinflammation, Biomedical and Biological Reports, Journal of Neurology and Epidemiology, American Journal of Stem Cells, and Clinical Immunology and Research.

\section{MICROGLIA CELLS}

Microglia cells are antigen presenting cells with myeloid origin that constituted approximately $10 \%$ of all glial cells of the adult mouse brain..$^{[1]}$ In the early embryo, the microglial precursors are located in the yolk sac and progressively migrate throughout the primitive brain. ${ }^{[2]}$ In the adult brain, some of microglial cells derive from the bone marrow, but this process only takes place when the brain is lesioned..$^{[1]}$ Microglial cells can display 4 morphological and functional stages: (1) resting microglia; (2) active microglia; (3) phagocytic microglia; and (4) senescent microglia. ${ }^{[3]}$ One of the functions of microglia is to provide the cell-mediated immunity response against pathogens by releasing chemokines or cytokines. ${ }^{[1]}$ However, the immune response and debris removing are not the only functions of microglial cells. Recently, it has been identified that microglia cells regulate neuronal apoptosis during the early brain development and modulate synaptic function. Strikingly, recent evidence indicates that microglia cells can regulate neurogenesis in the adult brain.

\section{THE VENTRICULAR-SUBVENTRICULAR ZONE}

The adult mammalian brain possesses specialized regions, also referred to as niches that host stem cells with neurogenic potential. One of these niches is the ventricular-subventricular zone (V-SVZ), an 
epithelial layer located in the lateral walls of the lateral ventricles. ${ }^{[4]}$ In the adult V-SVZ, three cell populations have been identified: type-B cells, type-C cells and type-A cells. ${ }^{[5]}$ The putative neural stem cell (NSC) is the type-B cell, an astroglial cell that can be identified by the expression of the glial fibrillary acidic protein, glutamate aspartate transporter, brain lipid binding protein, platelet-derived growth factor receptor $\alpha$, CD133, Id1, Tailless, vascular cell adhesion molecule 1, epidermal growth factor receptor (EGFR), and others. ${ }^{[4,5]}$ The activation of $\mathrm{B} 1$ cells depends on signaling pathways including sonic hedgehog, wingless-related integration site, Notch, bone morphogenetic proteins, ephrins, retinoic acid, betacellulin, stromal derived factor-1, pigment epithelium-derived factor and some intrinsic signals (Peroxiredoxin 1, sulfur oxide 2, arsenic-resistance 2, scute homolog 1, neuron-glia 2, Oligodendrocyte lineage transcription factor 2). ${ }^{[4,5]}$ After activation, type-B1 cells produce transit-amplifying progenitors (type-C cells) that express the EGFR and the transcription factors DIx2 and Mash1. ${ }^{[5,6]}$ Type-C cells divide and give rise to neuroblasts (type-A cells)

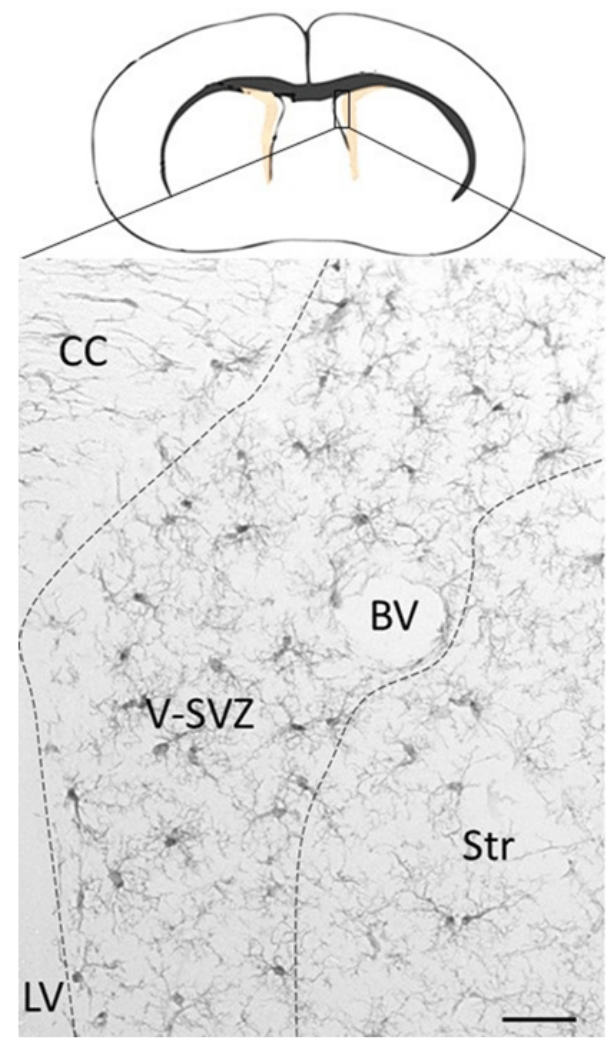

Figure 1: Microglia cells in the adult V-SVZ of mouse brain. Schematic bran section: The adult V-SVZ is the neurogenic niche lining the lateral walls of the lateral ventricles (LV). Photomicrography: Microglia cells labeled with anti-lba1 antibodies and revealed with $3,3^{\prime}$-Diaminobenzidine (DAB) technique. At resting stage, these cells exhibit ramified cell morphology and numerous thin processes. Note that microglia cells are more abundant in the V-SVZ as compared to adjacent brain regions: corpus callosum (CC) and striatum (Str). These cells are also in close contact with blood vessels (BV). Bar $=20 \mu \mathrm{m}$ that migrate to the olfactory bulb through the rostral migratory stream and become mature interneurons.

\section{THE RELATIONSHIP BETWEEN MICROGLIA CELLS AND THE V-SVZ}

In the adult brain microglia cells are present all along the V-SVZ [Figure 1] and remain in an intimate contact with niche cells. ${ }^{[7]}$ The first interaction between the V-SVZ and microglia begins when microglia cells begin to populate the embryonic brain. At the early stages of brain development an excessive number of neurons are produced. This surplus of neurons needs to be eliminated and microglia cells are the responsible effectors of that function. Thus microglial cells are crucial to maintain the balance of neurons and normal postnatal brain development.

In the adult V-SVZ microglia cells stimulate neurogenesis by releasing soluble factors within the niche ${ }^{[5]}$ This is a very complex process that requires the molecular feedback between NSCs and microglia cells, which release and express a myriad of molecules, such as: CD200, vascular endothelial growth factor, transforming growth factor $\beta$ in NSCs and CD200R, reactive nitrogen species/reactive oxygen species, insulin-like growth factor 1 , tumour necrosis factor- $\alpha$, Toll-like receptor-9, chemokine fractalkine, chemokine fractalkine receptor (CX3CR1), adenosine triphosphate-sensitive potassium channel IL-1 $\beta$, leukemia inhibitory factor, interferon- $\gamma . .^{[7,8,9]}$ In the V-SVZ, microglia cells presents certain degree of activation level and constantly release cytokines and neurotrophic factors with respect to other brain areas. This phenomenon suggests that NSCs are regulated by microglia cells. All of these events occur under physiological conditions, but under pathological circumstances these signals can be magnified. After activation, microglia cells enter into a phagocytic state to remove cell debris and damaged. Phagocytic microglia releases neurotrophic factors and cytokines that activate NSCs, thus trigger cell survival and neural regeneration after lesion. Microglial phagocytosis is one of the principal mechanisms to regulate and preserve the homeostasis in the production of neural progenitors in the postnatal brain. Microglia also eliminates aberrant cells that might later give rise to malignant cells or brain tumors.

\section{THE V-SVZ/ROSTRAL MIGRATORY STREAM MICROGLIA AND THEIR ROLE IN THE V-SVZ}

In a recent report, Xavier et al. ${ }^{[10]}$ demonstrate the presence of a microglial subpopulation in the V-SVZ 
and the rostral migratory stream (RMS) of CX3CR1enhanced green fluorescent protein mice, which have a locus of the fractalkine receptor CX3CR1 replaced by the gene encoding green fluorescent protein. They found a subpopulation of microglia (V-SVZI RMS microglia) that displayed distinct morphologies depending on the zone where they reside. The authors observed that some cells of this V-SVZ/RMS microglia presented an alternatively activated macrophage phenotype and released IL-4 and IL-10 cytokines via signal transducer and activator of transcription 6 phosphorylation.

The authors suggested that the differences in morphologies respect other brain areas may be due to a lower expression of purinergic receptors P2RY12, P2RY6, and P2RY1. Purinergic receptors use ATP for their activation and appear to regulate the processes and phagocytosis in microglial cells at non-neurogenic regions. In their study, Xavier et al. ${ }^{[10]}$ found that P2YR12 expression was virtually absent in the V-SVZ/RMS compared with the surrounding areas. Interestingly, the P2RY6 purinergic receptor, involved in phagocytosis process, is rarely expressed in the V-SVZ/RMS, which suggests that phagocytic events are not frequent in the V-SVZ and RMS. Conversely, P2RY1 expression is highly expressed in the V-SVZI RMS region in migrating neuroblasts, reaffirming the importance of P2RY1 in the migratory process of new neurons. Ablation of microglia increases the number of cells Ki67+ and BrdU+ in the V-SVZ/RMS and disrupts neuroblast migration. This evidence suggests that microglia play critical roles in neuroblast survival and migration in the V-SVZ.

In summary, microglial cells are the most important immune-cell mediator in the adult brain that preserve tissue homeostasis, regulate synaptic pruning, and sculpt neural circuitry. These cells are important components of the V-SVZ and RMS that play key roles in neurogenesis, neural survival and cell migration. Studying the relationship between NSCs and microglia cells is important for understanding the homeostatic mechanism that control the V-SVZ neurogenenic niche and surrounding areas. Nonetheless, this neural-immune interaction is still not well understood.
Thus, further studies are needed for clarifying the molecular events implied in the neurogenic process and determining its clinical relevance.

\section{Financial support and sponsorship}

OGP was financed by grants from Consejo Nacional de Ciencia y Tecnología (CONACyT-2015-01-465) and ELD by CONACyT's Fellowhip grant (No. 736004).

\section{Conflicts of interest}

There are no conflicts of interest.

\section{Patient consent}

No patient involved.

\section{Ethics approval}

This article does not contain any studies with human participants or animals.

\section{REFERENCES}

1. Greter M, Merad M. Regulation of microglia development and homeostasis. Glia 2013;61:121-7.

2. Ginhoux F, Greter M, Leboeuf M, Nandi S, See P, Gokhan S, Mehle MF, Conway SJ, Ng LG, Stanley ER, Samokhvalov IM, Merad M. Fate mapping analysis reveals that adult microglia derive from primitive macrophages. Science 2010;330:841-5.

3. Streit WJ, Xue QS. Life and death of microglia. J Neuroimmune Pharmacol 2009;4:371-9.

4. Lim DA, Alvarez-Buylla A. Adult neural stem cells stake their ground Trends Neurosci 2014;37:563-71.

5. Lim DA, Alvarez-Buylla A. The Adult Ventricular-Subventricular Zone (V-SVZ) and Olfactory Bulb (OB) Neurogenesis. Cold Spring Harb Perspect Biol 2016;8(5).

6. Doetsch F, Petreanu L, Caille I, Garcia-Verdugo JM, Alvarez-Buylla A. EGF converts transit-amplifying neurogenic precursors in the adult brain into multipotent stem cells. Neuron 2002;36:1021-34.

7. Gonzalez-Perez O, Gutierrez-Fernandez F, Lopez-Virgen V, CollasAguilar J, Quinones-Hinojosa A, Garcia-Verdugo JM. Immunological regulation of neurogenic niches in the adult brain. Neuroscience 2012;226:270-81

8. Su P, Zhang J, Zhao F, Aschner M, Chen J, Luo W. The interaction between microglia and neural stem/precursor cells. Brain Res Bull 2014;109:32-8.

9. Shigemoto-Mogami Y, Hoshikawa K, Goldman JE, Sekino Y, Sato K Microglia enhance neurogenesis and oligodendrogenesis in the early postnatal subventricular zone. J Neurosci 2014;34:2231-43.

10. Ribeiro Xavier AL, Kress BT, Goldman SA, Lacerda de Menezes JR, Nedergaard M. A distinct population of microglia supports adult neurogenesis in the subventricular zone. J Neurosci 2015;35:11848-61. 